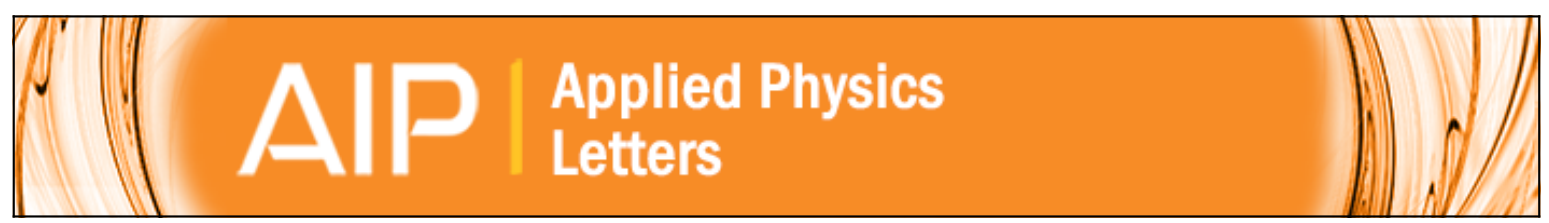

\title{
Polarization retention of thin ferroelectricya capacitors
}

Youn Jung Park, Jiyoun Chang, Seok Ju Kang, and Cheolmin Park

Citation: Applied Physics Letters 95, 102902 (2009); doi: 10.1063/1.3216053

View online: http://dx.doi.org/10.1063/1.3216053

View Table of Contents: http://scitation.aip.org/content/aip/journal/apl/95/10?ver=pdfcov

Published by the AIP Publishing

\section{Articles you may be interested in}

Polarization behavior of poly(vinylidene fluoride-trifluoroethylene) copolymer ferroelectric thin film capacitors for nonvolatile memory application in flexible electronics

J. Appl. Phys. 108, 094102 (2010); 10.1063/1.3500428

Polarization switching characteristics of $\mathrm{BiFeO} 3$ thin films epitaxially grown on $\mathrm{Pt} / \mathrm{MgO}$ at a low temperature Appl. Phys. Lett. 95, 242902 (2009); 10.1063/1.3275012

Polarization reversal kinetics of a lead zirconate titanate thin-film capacitor for nonvolatile memory J. Appl. Phys. 88, 3445 (2000); 10.1063/1.1288010

Downscaling of $\mathrm{Pb}(\mathrm{Zr}, \mathrm{Ti}) \mathrm{O} 3$ film thickness for low-voltage ferroelectric capacitors: Effect of charge relaxation at the interfaces

J. Appl. Phys. 88, 2154 (2000); 10.1063/1.1305854

Switching properties of $\mathrm{Pb}(\mathrm{Nb}, \mathrm{Zr}$, Ti)O 3 capacitors using SrRuO 3 electrodes Appl. Phys. Lett. 75, 1787 (1999); 10.1063/1.124820

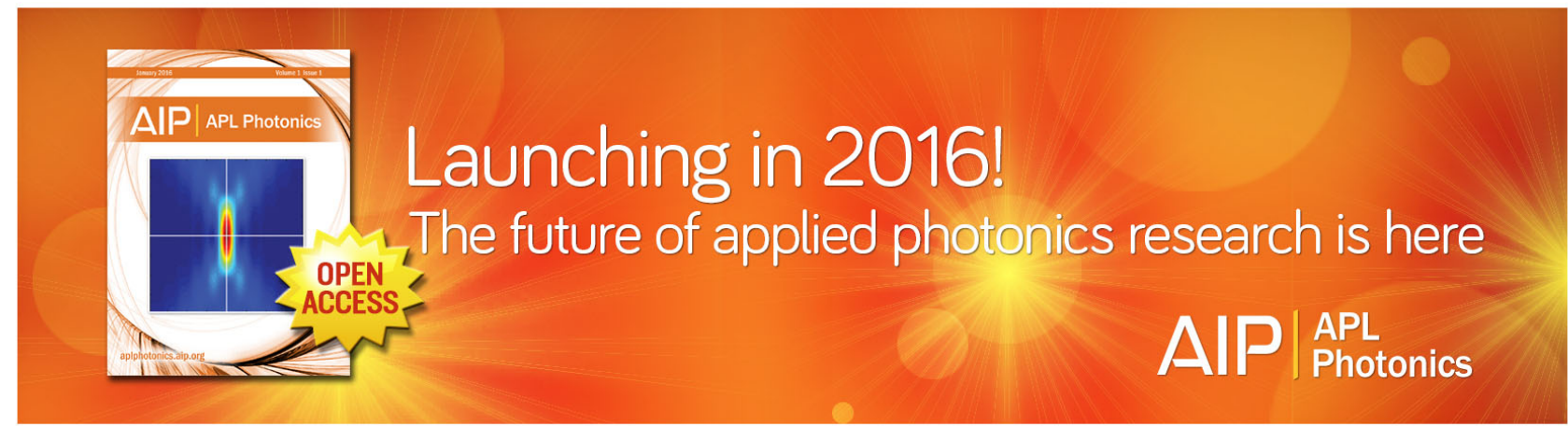




\title{
Polarization retention of thin ferroelectric poly(vinylidene fluoride-co-trifluoroethylene film capacitors
}

\author{
Youn Jung Park, Jiyoun Chang, Seok Ju Kang, and Cheolmin Park ${ }^{\mathrm{a})}$ \\ Department of Materials Science and Engineering, Yonsei University, Seoul 120-749, Republic of Korea
}

(Received 15 April 2009; accepted 1 August 2009; published online 9 September 2009)

\begin{abstract}
Excellent retention of the initial remanent polarizations was observed in ca. $200 \mathrm{~nm}$ thick ferroelectric poly(vinylidene fluoride-co-trifluoroethylene) film capacitors with the writing pulse amplitude and time width of $\pm 20 \mathrm{~V}$ and $1 \mathrm{~ms}$, respectively, over $200 \mathrm{~h}$ at $80{ }^{\circ} \mathrm{C}$. The opposite state program turned out more sensitive to retention deterioration than the same state one in both switching and nonswitching mode when either writing pulse amplitude or time width decreases. Nonswitching retention in the opposite state mode is in particular one of the most critical properties for designing a ferroelectric polymer capacitor memory. (C) 2009 American Institute of Physics. [doi:10.1063/1.3216053]
\end{abstract}

The great need to handle the information in various mobile applications attracts non volatile organic memories with competitive price and flexibility. ${ }^{1-3}$ The important candidate materials for memory devices are ferroelectric polymers such as poly(vinylidene fluoride) (PVDF) and its copolymers with trifluoroethylene (TrFE), embedded as thin films in either capacitors or transistors with their bistable polarization originating from electric dipoles in their molecular structure.

The long term reliability of ferroelectric memory devices is one of the most important requirements. Although the long duration properties such as retention, fatigue, imprint is considerably related to the memory failure itself, these problems in polymer ferroelectrics have been rarely explored, except the several works on fatigue properties in PVDF-TrFE films. ${ }^{4-6}$ In particular, data retention, defined by the ability to store the written state for certain period of time, is essential for successful realization of polymer memory devices. In spite of a number of the retention studies in inorganic ferroelectric thin film capacitors, ${ }^{7,8}$ only a few studies have been performed to characterize polarization retention loss in PVDF-TrFE thin film capacitors. In this contribution, therefore, we report on the polarization retention reliability of thin PVDF-TrFE film capacitors by measuring the retained switching and nonswitching polarization as a function of the baking time in various programmed test sequences.

Thin PVDF-TrFE (MSI sensors, PA, USA, PVDF: TrFE=72.5:27.5) films were spin coated on the Al substrate from 3 wt \% 2-butanone solution. Thickness of the fabricated films, measured by ellipsometry (Gaertner Scientific Co. USA), was approximately $200 \mathrm{~nm}$. The top Al electrodes with a unit area of $3.14 \times 10^{-4} \mathrm{~cm}^{2}$ were deposited by thermal evaporation using shadow masks on a polymer film with the pressure and the rate of $\sim 10^{-6} \mathrm{mB}, 0.1-0.2 \mathrm{~nm} / \mathrm{s}$, respectively. The polarization $(P)$-voltage $(V)$ hysteresis loops and retention profiles were recorded by a virtual ground circuit (Radiant Technologies Precision LC unit) in dark box at room temperature.

The retention characteristics were obtained by measuring the time-dependent changes of switched $\left(P^{*}\right)$ and nonswitched $\left(P^{\wedge}\right)$ polarization, respectively, by applying the pro-

\footnotetext{
a) Author to whom correspondence should be addressed. Electronic mail: cmpark@yonsei.ac.kr. Tel.: 02-2123-2833. FAX: 02-312-5375.
}

grammed pulses, as shown in Fig. 1(a). After a period during which the sample was held at the state preset by an initial writing pulse, the second pulse switches the sample to the other state and measures the switched polarization $\left(P^{*}\right)$. When the second pulse was applied with the same direction of a preset written pulse, we can measure the nonswitched polarization $\left(P^{\wedge}\right) .^{9}$ Each $P^{*}$ and $P^{\wedge}$ is characterized by two different test modes: same state (SS) and opposite state (OS) ${ }^{10}$ Left two pulses are for the SS retention and the other ones for the OS retention in Fig. 1(a). The SS retention tests evaluate the retaining ability of a previously given logic state. The reading pulse applied at room temperature measures either switched $\left(P_{\mathrm{SS}}^{*}\right)$ or non-switched $\left(P_{\mathrm{SS}}^{\wedge}\right)$ retained polarization. On the other hand, the OS retention test measures how much a given amounts of polarization is maintained after an additional writing voltage is given, opposite to a previous input stored for a long time. A device with an

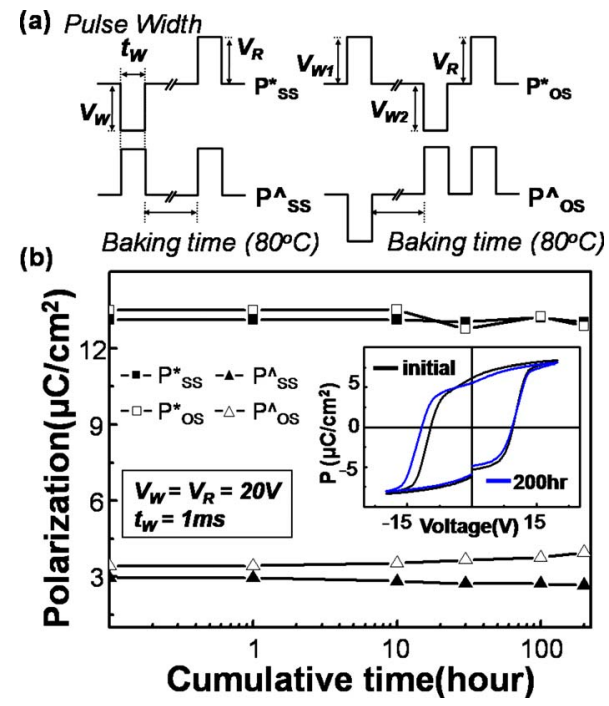

FIG. 1. (Color online) (a) Four sequences of programmed test pulses for the characterization of retention properties, switched $\left(P^{*}\right)$ and nonswitched $\left(P^{\wedge}\right)$ polarization of the SS and the OS retention. (b) The time dependent variations of switching polarization values $\left(P_{\mathrm{SS}}^{*}\right.$ and $\left.P_{\mathrm{OS}}^{*}\right)$ and nonswitching ones $\left(P_{\mathrm{SS}}^{\wedge}\right.$ and $\left.P_{\mathrm{OS}}^{\wedge}\right)$ in Al/PVDF-TrFE(200 nm)/Al capacitors, with the pulse width and amplitude of $1 \mathrm{~ms}, \pm 20 \mathrm{~V}$, respectively. The inset shows $P-V$ hysteresis loops obtained from a capacitor before and after the retention test performed at $80{ }^{\circ} \mathrm{C}$ for $200 \mathrm{~h}$. 
input voltage $V_{W 1}$ is baked for a certain time before which the second writing pulses of $V_{W 2}$ is delivered to induce the logic state opposite to $V_{W 1}$. Final reading pulses $\left(V_{R}\right)$ were subsequently applied after $30 \mathrm{~s}$ to measure $P_{\mathrm{OS}}^{*}$ and $P_{\mathrm{OS}}^{\wedge}$. The $V_{W 2}$ in OS measurement does not frequently fully restore the polarization formed by the input $V_{W 1}$ possibly due to imprint of a capacitor. This test is, however, different from the typical imprint test since no dc bias was applied. The retention loss in OS measurement already reported in PZT ferroelectrics is much more serious issue for practical applications and it is related to imprint arising from the growth of internal field. ${ }^{7,11}$ The width of all the reading pulses was $1 \mathrm{~ms}$, and the writing pulse width was varied from $1,5,10 \mathrm{~ms}$. To accelerate retention failures, ${ }^{9}$ we baked the polymer thin film capacitors at $80{ }^{\circ} \mathrm{C}$ for a certain period of time $(t)$, after applying writing pulses. The initial measurement after $0.1 \mathrm{~h}$ baking showed no polarization difference from the initial reference values.

Figure 1(b) shows the time dependent variations of switching and non-switching polarization values in $\mathrm{Al} /$ PVDF-TrFE $(200 \mathrm{~nm}) / \mathrm{Al}$ capacitors with the $1 \mathrm{~ms}$ and writing pulse of $\pm 20 \mathrm{~V}$. A $P$ - $V$ hysteresis loop obtained in an Al/PVDF-TrFE (200 nm)/Al capacitor exhibits the remanent polarization $\left(P_{r}\right)$ and the saturated maximum polarization of approximately \pm 6 and $\pm 8.2 \mu \mathrm{C} / \mathrm{cm}^{2}$, respectively, as shown in the inset of (b). While the baking time increases to $200 \mathrm{~h}$, both $P_{\mathrm{SS}}^{*}$ and $P_{\mathrm{SS}}^{\wedge}$ maintains approximately $99 \%$ of their initial polarization values as shown in Fig. 1(b). The polarization difference between $P_{\mathrm{SS}}^{*}$ and $P_{\mathrm{SS}}^{\wedge}$ in SS retention $\left(\Delta P_{\mathrm{SS}}\right)$ is closely related to the memory sensing margin and remains almost unchanged, which indicates the excellent retention properties of our ferroelectric polymer capacitors in the SS test. On the other hand, the polarization retention of the OS test is slightly altered in both switched and nonswitched mode. As a result, the polarization difference in OS retention $\left(\Delta P_{\mathrm{OS}}\right)$ marginally decreases with the baking time and $88.5 \%$ of its original value was left after $200 \mathrm{~h}$ baking.

Our results of the retention loss in the OS greater than the SS are qualitatively consistent with those observed in PZT and SBT. ${ }^{7-9}$ For the OS retention, which can be related to imprint of our capacitor, $\Delta P_{\mathrm{OS}}$ as a function of time in logarithm scale shows a linear dependence following the equation of $P(t)=P_{0}-m \log \left(t / t_{0}\right)$, where $t$ is time, $P_{o}$ is the polarization at $t=t_{0}$, and $m$ can be interpreted as a decay rate. ${ }^{12}$ The $m$ value of our PVDF-TrFE capacitor is approximately 0.358 , slightly larger than that of SBT of approximately $0.24 .^{9}$ Although a representative $P-V$ hysteresis loop for $\mathrm{Al} / \mathrm{PVDF}-\mathrm{TrFE} / \mathrm{Al}$ capacitor acquired after the $200 \mathrm{~h}$ retention tests is similar to one initially obtained before the test, the slight decrease of $P_{r}$ is apparent with the baking temperature at $80^{\circ} \mathrm{C}$ in the inset of Fig. 1(b). There is a slight increase of $-V_{c}$ after the retention test probably due to charge impurities accumulated on the PVDF-TrFE/Al interface as observed in several inorganic ferroelectrics. ${ }^{13}$

We also investigated the retention of the input polarization as a function of both the pulse amplitude and width of the writing voltage. Three different amplitudes of 15,18 , and $20 \mathrm{~V}$ with a constant pulse width $(1 \mathrm{~ms})$ were applied to measure the retention properties in the SSs and OSs plotted in Fig. 2. For both two state retention tests programmed with $18 \mathrm{~V}$, the retention characteristics of a capacitor are very similar to those observed with $20 \mathrm{~V}, 11 \%$ and $10 \%$ decrease

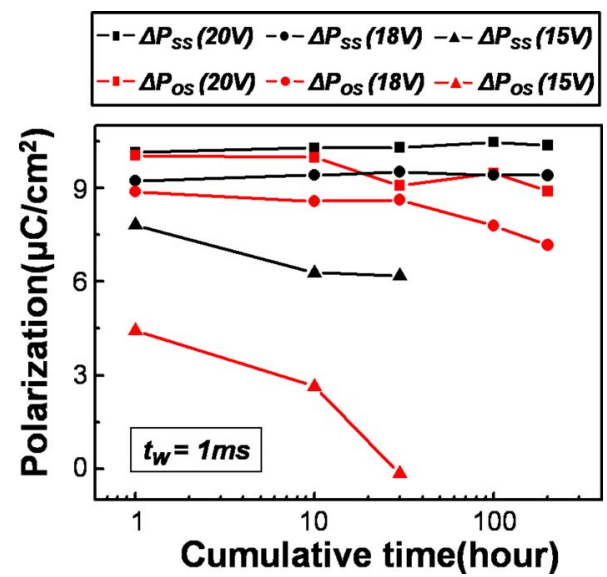

FIG. 2. (Color online) The baking time dependent variations of the SS polarization difference $\left(\Delta P_{\mathrm{SS}}\right)$ and the OS one $\left(\Delta P_{\mathrm{OS}}\right)$ between switching and non-switching in Al/PVDF-TrFE $(200 \mathrm{~nm}) / \mathrm{Al}$ capacitors with the $1 \mathrm{~ms}$ writing pulse of $\pm 20, \pm 18$, and $\pm 15 \mathrm{~V}$, respectively.

from the original polarization in the OS test was detected after $200 \mathrm{~h}$ baking with the applied voltage of 20 and $18 \mathrm{~V}$, respectively. The retained polarizations, however, drops dramatically with the cumulative time passed when the applied pulse is $15 \mathrm{~V}$ in both state modes. In the case of the SS, the polarization values were reduced to $80.4 \%$ and $79.1 \%$ of the pristine polarization after 10 and $30 \mathrm{~h}$ baking, respectively. More severely, for the OS, we observed the significant deterioration of the polarization value after $30 \mathrm{~h}$ baking from $4.5 \mu \mathrm{C} / \mathrm{cm}^{2}$ to almost zero. In the thin PVDF-TrFE film with unsaturated polarization at the programmed voltage amplitude of $15 \mathrm{~V}$, the poled $H-F$ dipoles in the written state became very unstable and almost completely depolarized with the baking time. Our results suggest that complete polarization saturation is essential for the good retention of the polarization in a ferroelectric PVDF-TrFE film.

Based on the results that the significant degradation of the polarization value occurred with the applying voltage and pulse width of $15 \mathrm{~V}$ and $1 \mathrm{~ms}$, respectively, we monitored the polarization decay by applying the voltage with different pulse width, $t_{w}$, of 5 and $10 \mathrm{~ms}$ at the amplitude of $15 \mathrm{~V}$. Contrary to the significant deterioration of polarization behavior with the pulse width of $1 \mathrm{~ms}$ (Fig. 2), $P_{\mathrm{SS}}^{*}$ and $P_{\mathrm{SS}}^{\wedge}$ after $200 \mathrm{~h}$ baking maintains $98 \%$ of their initial polarization values when the voltage train with $5 \mathrm{~ms}$ pulse width was employed as shown in Fig. 3(a). Consequently, the $\Delta P_{\mathrm{SS}}$ sustains its pristine amount after the $200 \mathrm{~h}$ passed. For the OS retention, however, approximately $8 \%$ decrease of $P_{\mathrm{OS}}^{*}$ and $35 \%$ increase of $P_{\mathrm{OS}}^{\wedge}$ results in the $27 \%$ decrease in the net polarization of $\Delta P_{\mathrm{OS}}$ after the $200 \mathrm{~h}$ baking. The significant improvement of $\Delta P_{\mathrm{OS}}$ with the pulse width of $5 \mathrm{~ms}$ compared to that with $1 \mathrm{~ms}$ may be attributed to the more fully saturated polarization resulting from the longer programing time.

The more slight improvement of polarization retention was made with $10 \mathrm{~ms}$ pulse width as shown in Fig. 3(b). Even with the low applied voltage of $15 \mathrm{~V}$, approximately $25 \%$ retention loss was observed with $10 \mathrm{~ms}$ pulse width in the OS test. Our results clearly show that the magnitude of retained polarization was affected by the pulse width and the significant degradation behavior occurred in the OS retention tests in ferroelectric polymer capacitor, consistent with the results in inorganic materials. 12,14 In both SS and OS test the 

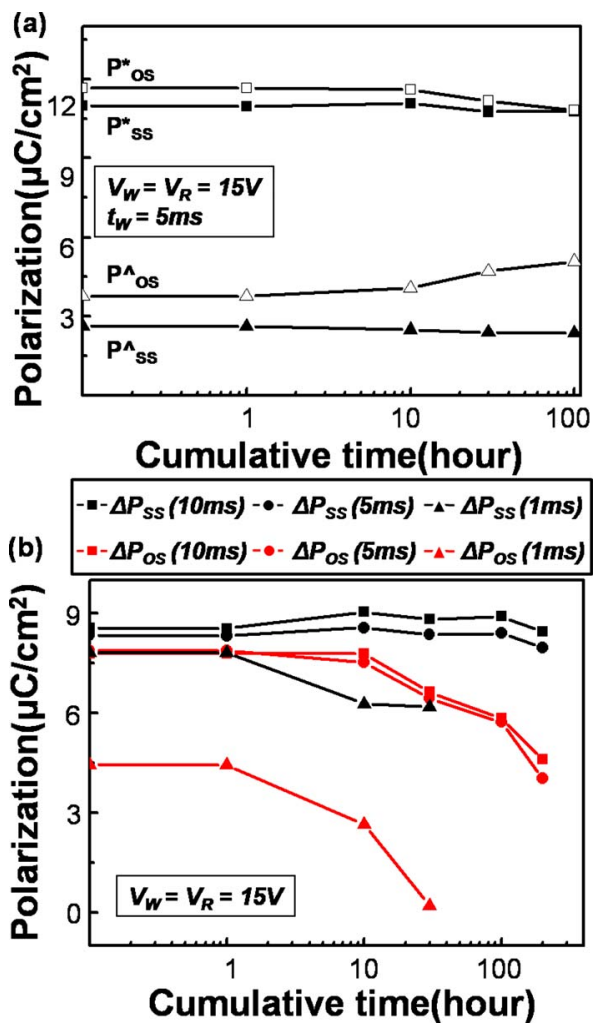

FIG. 3. (Color online) (a) The time dependent variations of switching polarization values $\left(P_{\mathrm{SS}}^{*}\right.$ and $\left.P_{\mathrm{OS}}^{*}\right)$ and nonswitching ones $\left(P_{\mathrm{SS}}^{\wedge}\right.$ and $\left.P_{\mathrm{OS}}^{\wedge}\right)$ in $\mathrm{Al} / \mathrm{PVDF}-\mathrm{TrFE}(200 \mathrm{~nm}) / \mathrm{Al}$ capacitors with the pulse width and amplitude of $5 \mathrm{~ms}, \pm 15 \mathrm{~V}$, respectively. (b) The time dependent variations of the polarization difference between switching and nonswitching mode in both same $\left(\Delta P_{\mathrm{SS}}\right)$ and $\mathrm{OS}\left(\Delta P_{\mathrm{OS}}\right)$ with the pulse widths of 1,5 , and $10 \mathrm{~ms}$.

reduced $\Delta P_{\mathrm{SS}}$ and $\Delta P_{\mathrm{OS}}$ at the low voltage of $15 \mathrm{~V}$ were more attributed to the deterioration of nonswitching retention than that of switching.

The decay rates $(m)$ of the retention loss in the OS tests were calculated from Fig. 2 and 3 and are plotted as a function of both pulse amplitude and width of the applied writing voltage in Fig. 4. As expected, the decay rates rapidly increase with the decrease of both pulse amplitude and width. The maximum and minimum $m$ values of our PVDF-TrFE capacitors are approximately 1.75 and 0.36 , respectively.

In summary, the retention loss of Al/PVDF-TrFE/Al capacitors was monitored with four kinds of pulse trains in order to characterize both switched and nonswitched polarization for each SS and OS modes. Almost complete retention of the initial remanent polarization in the same and opposite modes at $80^{\circ} \mathrm{C}$ over $200 \mathrm{~h}$, respectively, was observed at $\pm 20 \mathrm{~V}$ and $1 \mathrm{~ms}$ in writing pulse amplitude and time width, respectively. The retention was significantly deteriorated when both writing pulse amplitude and time width decrease. Nonswitching retention in the OS mode turned out

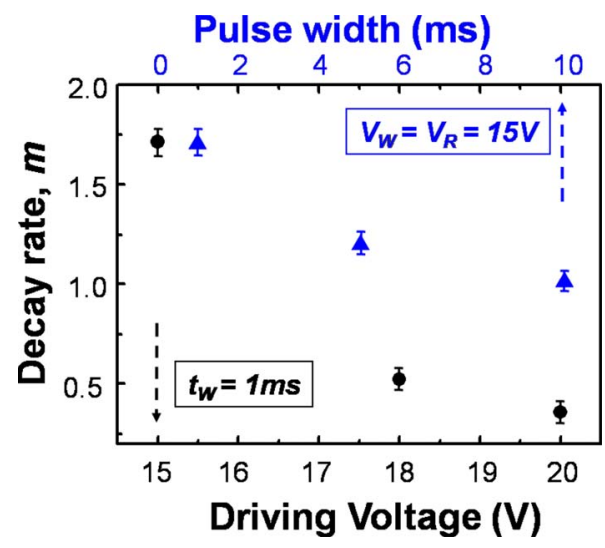

FIG. 4. (Color online) The plots of the decay rate $(\mathrm{m})$ as a function of the pulse width (top) and amplitude of the writing voltage (bottom). The decay rate dependence on the amplitude was measured with the pulse width of 1 $\mathrm{ms}$, and the other dependence on the pulse width was performed with the applying voltage of $15 \mathrm{~V}$.

one of the most important properties for realizing a nonvolatile ferroelectric polymer memory capacitor.

This project was supported by The National Research Program for Memory Development and the "SYSTEM2010" project sponsored by the Korea Ministry of Knowledge and Economy and Samsung Electronics, Co., Ltd. This work was supported by the Second Stage of Brain Korea 21 Project in 2006, the Seoul Science Fellowship, and the Korea Science and Engineering Foundation (KOSEF) grant funded by the Korea government (MEST) (Grant No. R11-2007-05003001-0).

${ }^{1}$ A. J. Lovinger, Science 220, 1115 (1983).

${ }^{2}$ R. C. G. Naber, P. W. M. Blom, A. W. Marsman, F. J. Touwslager, S. Setayesh, and D. M. de Leeuw, Nature Mater. 4, 243 (2005).

${ }^{3}$ Z. Hu, M. Tian, B. Nysten, and A. M. Jonas, Nature Mater. 8, 62 (2009).

${ }^{4}$ S. Sakai, M. Date, and T. Furukawa, Jpn. J. Appl. Phys., Part 1 41, 3822 (2002).

${ }^{5}$ G. Zhu, Z. Zeng, L. Zhang, and X. Yan, Appl. Phys. Lett. 89, 102905 (2006).

${ }^{6}$ G. Zhu, Z. Zeng, Q. Zhang, X. Yan, and R. Liu, J. Appl. Phys. 103, 084125 (2008).

${ }^{7}$ B. S. Kang, J.-G. Yoon, D. J. Kim, T. W. Noh, T. K. Song, Y. K. Lee, J. K. Lee, and Y. S. Park, Appl. Phys. Lett. 82, 2124 (2003).

${ }^{8}$ B. S. Kang, D. J. Kim, J. Y. Jo, T. W. Noh, J.-G. Yoon, T. K. Song, Y. K. Lee, J. K. Lee, S. Shin, and Y. S. Park, Appl. Phys. Lett. 82, 3127 (2004).

${ }^{9}$ Y. Shimada, K. Nakao, A. Inoue, M. Azuma, Y. Uemoto, E. Fujii, and T. Otsuki, Appl. Phys. Lett. 71, 2538 (1997).

${ }^{10}$ J.-S. Lee, B. S. Kang, and Q. X. Jia, Appl. Phys. Lett. 91, 142901 (2007)

${ }^{11}$ R. R. Mehta, B. D. Silverman, and J. T. Jacobs, J. Appl. Phys. 44, 3379 (1973).

${ }^{12}$ J. M. Benedetto, R. A. Moore, and F. B. Mclean, J. Appl. Phys. 75, 460 (1994).

${ }^{13}$ A. Q. Jiang, Y. Y. Lin, and T. A. Tang, Appl. Phys. Lett. 91, 202906 (2007).

${ }^{14}$ A. Gruverman and M. Tanaka, J. Appl. Phys. 89, 1836 (2001). 